\title{
Effect of Prenatal Steroids on Skin Surface Hydrophobicity in the Premature Rat
}

\author{
FELIX A. OKAH, WILLIAM L. PICKENS, AND STEVEN B. HOATH \\ Division of Neonatology, Children's Hospital Medical Center, and University of Cincinnati, \\ Cincinnati, Ohio 45267
}

ABSTRACT

\begin{abstract}
The skin surface of the newborn rat at term is highly hydrophobic. This surface hydrophobicity plays a putative role in the transition from an aqueous to a gaseous environment at birth and is dependent on the presence of an intact periderm. Glucocorticoids given to pregnant dams, during late gestation, will accelerate formation of the stratum corneum and reduce transepidermal water loss in prematurely delivered pups. We tested the related hypotheses that surface hydrophobicity and maturation of the periderm are developmentally accelerated by prenatal exposure to steroids. Thirty pregnant Sprague-Dawley rats received either normal saline or $0.5 \mathrm{mg} / \mathrm{kg}$ betamethasone on $\mathrm{d} 17$ of gestation. After cesarean delivery on d 18, 19, and 20, dorsal skin surface hydrophobicity was quantified by direct surface electrical capacitance (SEC) measurement. Initial skin surface hydration at birth was significantly lower in steroid-treated pups than in control pups at gestational ages 19 and $20 \mathrm{~d}(3060 \pm 1379$ versus $4441 \pm 153 \mathrm{pF}$ and $646 \pm 295$ versus $1493 \pm 1019 \mathrm{pF}$,
\end{abstract}

respectively, $p<0.001$, mean $\pm \mathrm{SD}$ ). Likewise, after desorption of amniotic fluid, baseline skin hydration was significantly lower in steroid-treated pups than in control pups at gestational ages 19 and $20 \mathrm{~d}(1862 \pm 1560 \mathrm{pF}$ versus $4278 \pm 97 \mathrm{pF}$ and $60 \pm 56 \mathrm{pF}$ versus $128 \pm 264 \mathrm{pF}, p<0.001)$. Scanning and transmission electron microscopy showed morphologic maturation of the periderm after steroid treatment. These results demonstrate accelerated development of both functional and structural correlates of skin surface hydrophobicity in the premature rat after prenatal exposure to steroids. (Pediatr Res 37: 402-408, 1995)

\[ \text { Abbreviations } \]
SEC, surface electrical capacitance
TEWL, transepidermal water loss
$\mathrm{C}_{\mathrm{bl}}$, baseline surface hydration
$\mathrm{C}_{\mathrm{pk}}$, peak skin surface hydration
$\mathrm{C}_{\mathrm{sl}}$, slope of moisture accumulation

Premature birth bodes ill for mammals. This fact is reflected in the disproportionate mortality among prematurely delivered human infants $(1,2)$. This disparity in survival between the premature and mature newborn is related to differences in the degree of development and function of various organs. One such organ is the skin. Postnatal studies of the ontogeny of water sorption-desorption indicate that the mature newborn mammal possesses a hydrophobic skin surface at the time of birth (3-5). A potential adaptive advantage of surface hydrophobicity is easy elimination of exogenous water from the skin surface (e.g. amniotic fluid), thereby reducing the amount of heat lost by evaporation. In humans, the ability to desorb topically applied water is at a peak in the immediate newborn period (4). Rats also possess a highly hydrophobic surface at birth. This hydrophobicity is gradually lost over the first $3 \mathrm{~d}$ of life (5).

Previous studies in newborn infants have focused on transepidermal water loss and have clearly shown that the preterm

Received September 30, 1994; accepted January 19, 1995

Correspondence and reprint requests: Steven B. Hoath, M.D., Division of Neonatology, Department of Pediatrics, University of Cincinnati Medical Center, 231 Bethesda Avenue, Cincinnati. $\mathrm{OH} 45267$ infant has an immature and ineffective epidermal barrier (6-9). This finding is important insofar as the premature human infant experiences major difficulties with body temperature regulation and infection. These problems relate, in part, to deficient epidermal barrier formation and contribute to the high morbidity and mortality observed in this high risk patient population. Clinically, epithelial maturation in other organs such as the lung is accelerated by prenatal administration of glucocorticoids to the mother (10). A recent report by Azterbaum et al. (11) indicates similar maturational effects occur in the epidermis of the premature rat.

In this study, we investigated the effect of prenatally administered steroids on skin surface hydrophobicity in the premature rat model. Skin surface hydrophobicity was determined as described previously using skin surface electrical capacitance measurements $(3,5)$. Specifically, we measured the peak surface hydration of the skin after delivery and the subsequent desorption of amniotic fluid from the skin surface (evaporative drying) under controlled environmental conditions. In addition, we obtained continuous skin surface capacitance recordings. During continuous measurement the capacitance value at $1 \mathrm{~s}$ provided a measure of baseline surface hydration, whereas the slope, by measuring the changing hydration of the skin surface 
occluded by the meter probe, provided an indirect measure of TEWL (12).

\section{METHODS}

Experimental design. The model for this study was a partially nested hierarchical design with one covariate (birth weight of pups). The dependent or outcome variables were 1) the peak skin surface hydration immediately after birth (a measure of hydrophobicity), 2) the baseline skin surface hydration (also a measure of skin surface hydrophobicity), and 3) the slope, $\mathrm{C}_{\mathrm{s}}$, of moisture accumulation during continuous capacitance measurement (a measure of TEWL) (5). Skin hydration was measured by bascline surface electrical capacitance. The independent variables included treatment (steroid versus vehicle), gestational age at birth, and the dam (from which a pup was delivered).

Animal model Sprague-Dawley rat. Thirty timed pregnant Sprague-Dawley rats (Zivic-Miller Laboratories Inc., Zelienople Park, PA) were obtained and randomly assigned to be delivered by cesarean section on $\mathrm{d} 18,19$, and 20 of gestation (term gestation in the Sprague-Dawley rat is 21-22 d). The dams were further randomized to one of two treatment groups to receive cither intramuscular injection of normal saline (vehicle) or betamethasone $(0.5 \mathrm{mg} / \mathrm{kg})$ on the 17 th $\mathrm{d}$ of gestation. This steroid dose was chosen because it was similar to currently administered doses in human adults. A table of random numbers was used for all assignments (13).

Before delivery, dams were anesthetized with Metofane (Pitman-Moore Inc., Washington Crossing, NJ). The uteri were cxposed via a midline longitudinal incision and the pups were delivered individually. The litter size varied from six to 17 pups. The characteristics of the groups of pups are shown in Table 1.

At birth, each pup was immediately wcighed and passed to a second investigator who transferred the pup to an incubator (Air Shields model C-100) and gently blotted the skin surface three times in rapid succession with dry cotton gauze sponges (Kendall Healthcare Products Company, Mansfield, MA). The initial surface electrical capacitance measurement was taken at approximately $15 \mathrm{~s}$ after delivery, and subsequent measurements were recorded from the dorsal skin surface at 10 -s intervals for $1 \mathrm{~min}$. This event was termed the desorption phase of the experiment. The first reading after blotting was termed the peak surface hydration, $\mathrm{C}_{\mathrm{pk}}$. This value is a measure of the quantity of water present on the skin surface immediately after birth and, particularly in the older pups, of the extent to which amniotic fluid is repelled from the skin surface (hydrophobicity) after birth.

After desorption measurements and the attainment of a baseline plateau, the probc head was reapplied to the dorsal skin surface, and continuous readings were obtained over a 10 -s period. The reading at $1-\mathrm{s}, \mathrm{C}_{\mathrm{b}, \mathrm{l}}$, was used as a measure of baseline surface hydration (after desorption of amniotic fluid), whereas the slope, $\mathrm{C}_{\mathrm{sl}}$, of the 10 -s recording provided a measure of the rate at which tissue water traversed the epidermal barrier to collect beneath the probe head. Thus, the continuous surface capacitance measurements provided an indirect measure of transepidermal water movement as previously shown in the nconatal rat model (12). The environmental temperature and relative humidity in the incubator were maintained at $33^{\circ} \mathrm{C}$ and $34 \%$, respectively.

Capacitance measurements. Surface electrical capacitance measurements provided a measure of surface hydration. These measurements were obtained using a battery-operated Dermaphase meter model 9003 (NOVA Technology Corporation, Gloucester, MA). This instrument has a spring-loaded probe head with a flat contact surface (diameter $=5 \mathrm{~mm}$ ) containing two concentric brass electrodes separated by a nonconducting resin. Skin capacitance was measured in picofarad equivalents and converted to picofarads as previously described $(3,5,12)$. Capacitance measurements were performed in a C-100 incubator (Air Shields, Hatboro, PA), and data were downloaded on line to a Compaq LTE LITE/20 laptop computer. Datafiles were subsequently transferred to a Hewlett Packard 486/66N computer for subsequent analysis using the SAS program.

Histologic evaluation. Histologic correlates of skin surface hydrophobicity and barrier function were examined by transmission and scanning electron microscopy. Specifically, we sought for evidence of accelerated periderm and/or stratum corneum development. For these studies, newborn rats were euthanized and fixed immediately in $2 \%$ glutaraldehyde and $2 \%$ paraformaldehyde in $0.1 \mathrm{M}$ sodium cacodylate $(\mathrm{pH} 7.4)$, postfixed with $1 \%$ osmium tetroxide in $0.1 \mathrm{M}$ sodium cacodylate buffer, and subsequently embedded in Epon-Araldite (Ciba-Gcigy, Summit, NJ $(14,15)$. Scctions of rat skin were examined by transmission clectron microscopy using the JEOL 100CX transmission electron microscope. Additional sections were critical-point dried and sputter-coated with gold-

Table 1. Litter sizes, mean body weights, and mean $C_{p k}$ of control and steroid-treated Sprague-Dawley pups delivered by cesarean section on gestational $d 18,19$, and 20

\begin{tabular}{|c|c|c|c|c|c|c|}
\hline & \multicolumn{2}{|c|}{ Day 18} & \multicolumn{2}{|c|}{ Day 19} & \multicolumn{2}{|c|}{ Day 20} \\
\hline & Control & Steroid & Control & Steroid & Control & Steroid \\
\hline No. of dams & 5 & 5 & 5 & 5 & 5 & 5 \\
\hline Mean wcight of pups (mg) & $\begin{array}{l}1673 \\
(159)\end{array}$ & $\begin{array}{l}1628 \\
(227)\end{array}$ & $\begin{array}{l}22.14 \\
(298)\end{array}$ & $\begin{array}{c}2467^{*} \\
(270)\end{array}$ & $\begin{array}{l}3880 \\
(484)\end{array}$ & $\begin{array}{l}4115^{*} \\
(344)\end{array}$ \\
\hline Mean $\mathrm{C}_{\mathrm{pk}}(\mathrm{pF})$ & $\begin{array}{r}4449 \\
(25)\end{array}$ & $\begin{array}{l}4399 \\
(136)\end{array}$ & $\begin{array}{l}4441 \\
(153)\end{array}$ & $\begin{array}{c}3060^{*} \\
(1380)\end{array}$ & $\begin{array}{c}1493 \\
(1019)\end{array}$ & $\begin{array}{c}646^{*} \\
(295)\end{array}$ \\
\hline
\end{tabular}

Data are: $n=377$ mean $\pm(\mathrm{SD})$.

$* p<0.05$. 
palladium for examination by scanning electron microscopy. The latter study was performed using the JEOL $35 \mathrm{~S}$ scanning electron microscope (Japanese Electron Optic Laboratories, Bedford, MA).

Data analysis. The variables for statistical comparison were the degree of peak skin surface hydration, $\mathrm{C}_{\mathrm{pk}}$, after delivery, the baseline surface hydration, $\mathrm{C}_{\mathrm{bl}}$, at the beginning of the continuous reading, and the slope of moisture accumulation, $\mathrm{C}_{\mathrm{sl}}$. Statistical analyses were performed using the PROC GLM function in the SAS program. Distribution of the residuals of the outcome variable was tested by the Shapiro-Wilks test. Paired comparisons of the various treatment combinations (gestational age and type of treatment) were performed by the Student-Newman-Keuls test for multiple comparisons. A $p$ value less than 0.05 was considered significant. All values were expressed as mean $\pm \mathrm{SD}$.

\section{RESULTS}

On $\mathrm{d} 18$, the mean peak surface capacitance values, $\mathrm{C}_{\mathrm{pk}}$, in steroid-treated and control pups were not significantly different from each other $(4399 \pm 136 \mathrm{pF}$ versus $4449 \pm 25 \mathrm{pF}$, respectively, $p>0.05$ ). In both groups, peak capacitance was high and barely changed during the desorption part of the study, i.e. the skin surface failed to dry (Fig. 1A). Baseline surface hydration, $\mathrm{C}_{\mathrm{bl}}$, at the beginning of the 10 -s continuous measurement was not significantly different in steroid-treated and control pups $(4238 \pm 96 \mathrm{pF}$ versus $4286 \pm 137 \mathrm{pF}$, respectively, $p>0.05$ ). Continuous surface capacitance measurements revealed flat profiles indicative of a maximally saturated (hydrated) surface $\left(\mathrm{C}_{\mathrm{s} L}=-1.8 \pm 6.3 \mathrm{pF} / \mathrm{s}\right.$ versus $-3.8 \pm 7.0 \mathrm{pF} / \mathrm{s}$, respectively, $p>0.05$ ) (Fig. $2 A$ ).

By d 19, the mean peak surface capacitance values, $C_{p k}$, were significantly lower in steroid-treated than in control pups (3060) $\pm 1380 \mathrm{pF}$ versus $4441 \pm 153 \mathrm{pF}$, respectively, $p<$ 0.01). Desorption (evaporative drying) was noted in both groups. However, this change in surface hydration was more prominent in steroid-treated than in control pups $(3060 \pm 1380$ $\mathrm{pF}$ to $1862 \pm 1560 \mathrm{pF}$ versus $4441 \pm 153 \mathrm{pF}$ to $4278 \pm 97 \mathrm{pF}$, respectively) (Figs. $1 B$ and $2 B$ ). As a result, the baseline surface hydration, $\mathrm{C}_{\mathrm{bl}}$, was significantly lower in the steroidtreated than in control pups $(1862 \pm 1560 \mathrm{pF}$ versus $4278 \pm$ $97 \mathrm{pF}$, respectively, $p<0.0001)$. During continuous recording, the skin of control pups registered an essentially flat response $\left(\mathrm{C}_{\mathrm{s} I}=0.5 \pm 6.9 \mathrm{pF} / \mathrm{s}\right)$ similar to the $\mathrm{d} 18$ control pups. Steroid-treated pups, however, demonstrated a significantly steeper slope $(153 \pm 95 \mathrm{pF} / \mathrm{s})$ indicative of moisture collection beneath the probe head (Fig. $2 B$ ).

By d 20, the $C_{p k}$ values were significantly lower in steroidtreated pups $(646 \pm 295 \mathrm{pF}$ versus $1493 \pm 1019 \mathrm{pF}$, respectively, $p<0.0001)$, and significant desorption was observed in both groups (Fig. $1 C$ ). Baseline surface hydration $(60 \pm 56 \mathrm{pF}$ versus $128 \pm 264 \mathrm{pF}$ ) and the slope of the continuous surface capacitance recordings $(20.3 \pm 8.0 \mathrm{pF} / \mathrm{s}$ versus $83.4 \pm 62.2$ $\mathrm{pF} / \mathrm{s}$ ) were consistent with a significantly better barrier after steroid treatment (Fig. 2C).

The model was tested on PROC GLM of SAS. For the model, $R_{2}=0.92, F=138, d f=30,346 ; p=0.0001$.
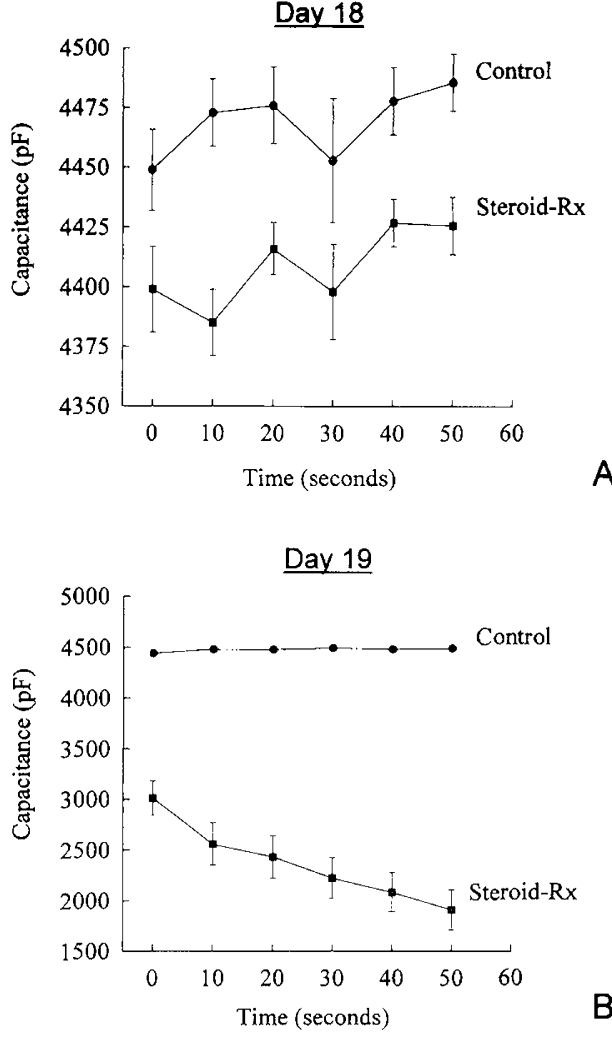

B

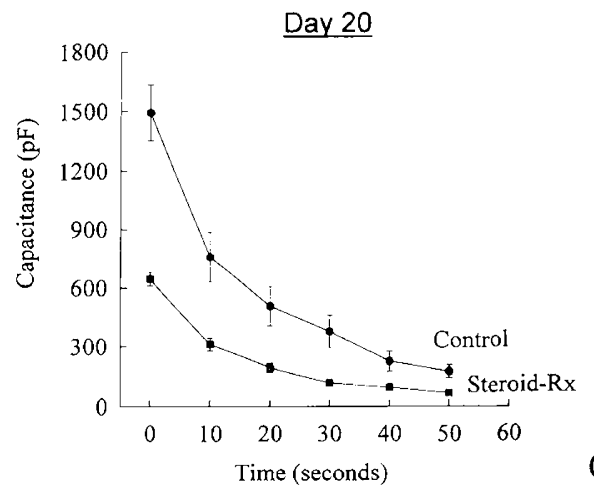

Figure 1. A-C, Effect of prenatal steroid administration to alter water sorption-desorption kinetics (hydrophobicity) from the dorsal skin surface of premature rats. A single dose of betamethasone $(0.5 \mathrm{mg} / \mathrm{kg})$ was given to the mother on gestational d 17. Pups were delivered by cesarean section on d 18 $(A), 19(B)$, and $20(C)$, placed in an incubator under controlled environmental conditions, and gently blotted with gauze. The dorsal skin was allowed to dry by desorption of amniotic fluid for 1 min during which time surface electrical capacitance measurements were obtained every $10 \mathrm{~s}$. Prenatal steroid treatment and advancing gestational age resulted in a progressively drier, more hydrophobic surface as indicated by the decrease in peak (time 0) capacitance measurements and the ability to desorb surface water resulting in low baseline (time 50 ) readings. SEC data arc reported as means \pm SEM. The total number of pups studied was 377

Furthermore, the analysis revealed that both $\mathrm{C}_{\mathrm{pk}}$ and $\mathrm{C}_{\mathrm{bl}}$ were related to the type of treatment received $(F=142.01$ and 597 , $d f=1,24 ; p<0.0001)$, gestational age at birth $(F=31.8$ and $111, d f=2,24 ; p<0.0001)$, weight of the pups $(F=20.12$ and 27, $d f=1,30 ; p<0.0001)$, the dams $(F=12.8$ and 59 , $d f=24,346 ; p<0.0001)$ and the interaction effect of age and 

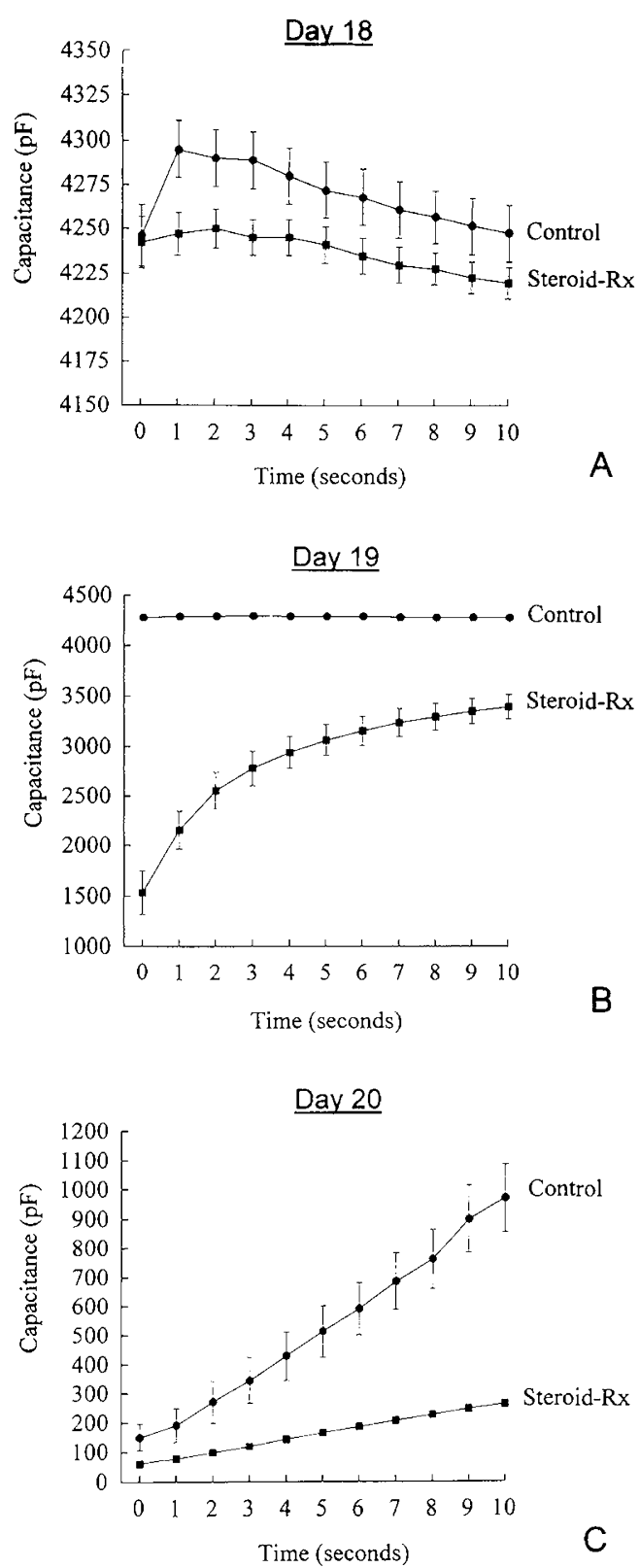

Figure 2. $A-C$, Effect of prenatal steroid administration to alter the rate of transepidermal water movement (barrier formation) from the dorsal skin of premature rats. After caesarean delivery, pups were blotted with gauze and allowed to dry by desorption of amniotic fluid for 1 min (see Fig. $1 A-C$ ). Subsequently, the SEC probc head was placed against the dorsal epidermal surface, and continuous SEC readings were recorded over a subsequent 10 -s period. On gestational d $18(A)$ there was little difference between treatment groups. On gestational d $19(B)$, steroid-treated pups exhibited a relatively low baseline (time 0) SEC followed by a rapid increase, whereas control pups exhibited a high SEC throughout the measurement interval, indicating a maximally hydrated surface and no effective barrier. On gestational d $20(C)$, both groups exhibited lower baseline SEC values (steroid group $<$ controls). Probe occlusion resulted in a more rapid increase in the control group (greater slope), indicating a less effective barricr to transepidermal water movement. $\mathrm{SEC}$ data are reported as means $\pm \mathrm{SEM}$.

treatment $(F=41.9$ and $682, d f=2,24 ; p<0.0001)$. The residuals of the dependent variables were not normally distributed, and because they were bimodally distributed, it was not possible to normalize the distribution of residuals within the model. For this reason, only $F$ values greater than 20 were considered to be significant (Chebyschev law of large numbers) by the parametric statistical methods used in this analysis (16). Thus, the primary factors that significantly influenced peak and baseline skin surface capacitance were treatment effect and gestational age with a small effect from birth weight. Of note, steroid-treated pups were significantly heavier than control pups by gestational ages $\mathrm{d} 19$ and $20(2467 \pm 270 \mathrm{mg}$ versus $2214 \pm 298 \mathrm{mg}$ and $4115 \pm 344 \mathrm{mg}$ versus $3880 \mathrm{mg} \pm$ $484 \mathrm{mg}$, respectively, $p<0.01)$. Steroid-treated and control pups on gestational d 18 showed no differences in birth weights $(1625 \pm 224 \mathrm{mg}$ versus $1673 \pm 159 \mathrm{mg}, p>0.05)$.

Transmission electron microscopic examination (Fig. 3) of the ultrastructural features of the outermost peridermal layer of the dorsal skin of premature pups on d 19 of gestation revealed a "blebbed" appearance characteristic of absorptive epithelia. The inner cellular layer of the periderm contained numerous dark staining granules, similar to those previously described in fetal mice by Chang et al. (17). Underlying the periderm in steroid-treated pups, there was a lamellar organization characteristic of early stratum corneum development. No cornification was observed in age-matched control pups. On scanning electron micrographs (Fig. 4) the peridermal surface of both steroid $(B)$ and control (A) pups exhibited a "cerebriform" appearance with multiple tissue folds containing superficial granules (diameter $=1-2 \mu \mathrm{m}$ ). However, in steroid-treated pups, the topography was relatively flat and featureless, particularly over the domes of the tissue folds, and the superficial granules were less distinct.

\section{DISCUSSION}

In this study, we have shown that prenatal administration of steroids accelerates maturation of the epidermal/peridermal complex in prematurely born rats. SEC measurements were used to measure skin hydration immediately after delivery and after a controlled period of evaporation. These measures allowed a quantitative assessment of the degree of surface hydrophobicity. Surface hydrophobicity was primarily influenced by prenatal exposure to steroids and the gestational age of the premature pups. The findings on ultrastructural studies using scanning and transmission electron microscopy support the hypothesis that surface hydrophobicity properties may be due to maturation of the periderm in the developing rat. Maturation of the periderm and the underlying stratum corneum occur concomitantly, however, and both structures are developmentally accelerated by prenatal steroid exposure. Therefore, a contribution from the underlying stratum corneum to surface hydrophobicity cannot be ruled out and would be expected insofar as a mature stratum corneum limits egress of water to the outermost surface layers.

The higher peak and baseline SEC readings observed in control pups at all ages examined indicate a more hydrated skin surface. This finding may be interpreted in two ways. First, it may indicate the presence of a hydrophobic surface that is incapable of adequately repelling amniotic fluid at the time of birth (inadequate hydrophobicity) and/or, second, it may be due to an inefficient barrier that allows egress of tissue water through the skin (diminished barrier to transepidermal water 


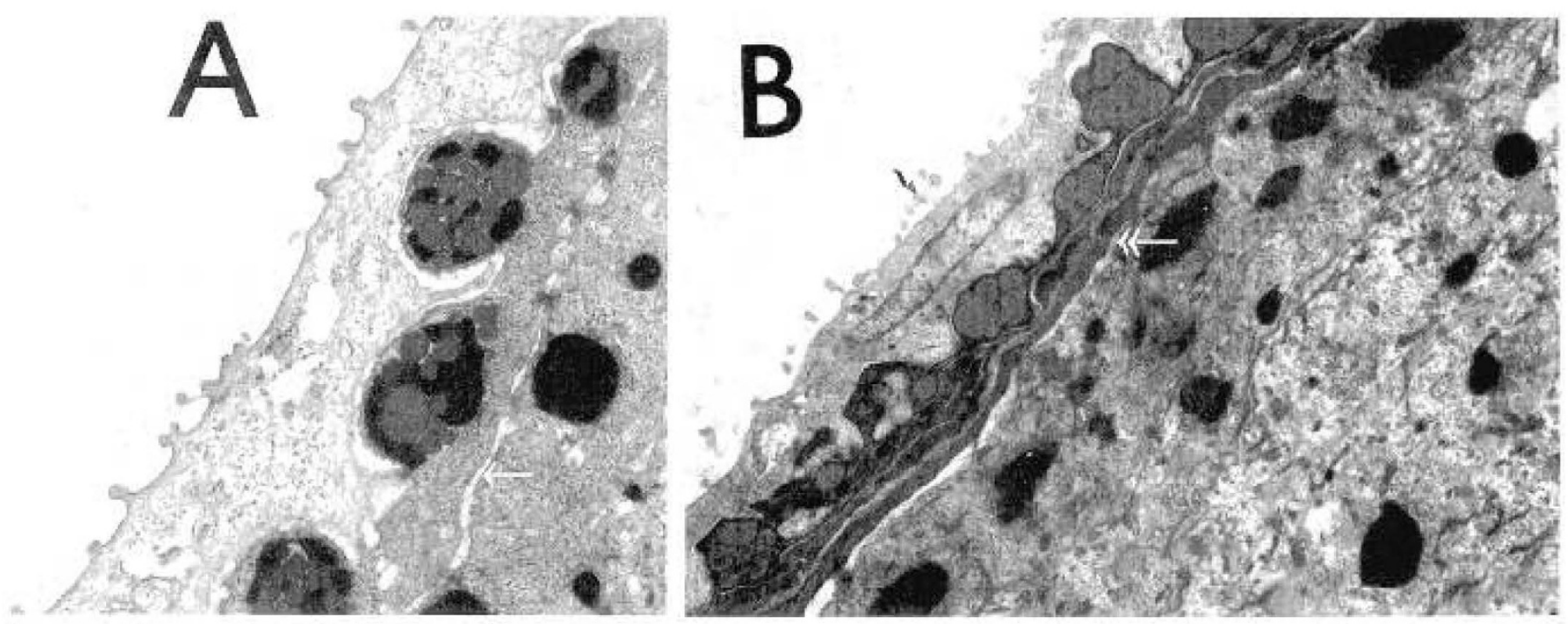

Figure 3. Ultrastructural examination of the epidermal/peridermal barrier in the premature rat by transmission electron microscopy. Photomicrographs of specimens from control $(A)$ and stcroid-treated $(B)$ dams collected on gestational d 19. The outermost surface of the pcriderm exhibits a "blebbed" appearance characteristic of an absorptive epithelium. The inner cellular layer of the periderm contains numerous dark staining granules. Note the lamellar organization characteristic of stratum corneum underlying the periderm in the steroid-treated specimen $(B$, arrow). No cornification has occurred in the age-matched control pups (A, arrow). Magnification: $A, \times 10000 ; B, \times 7200$.
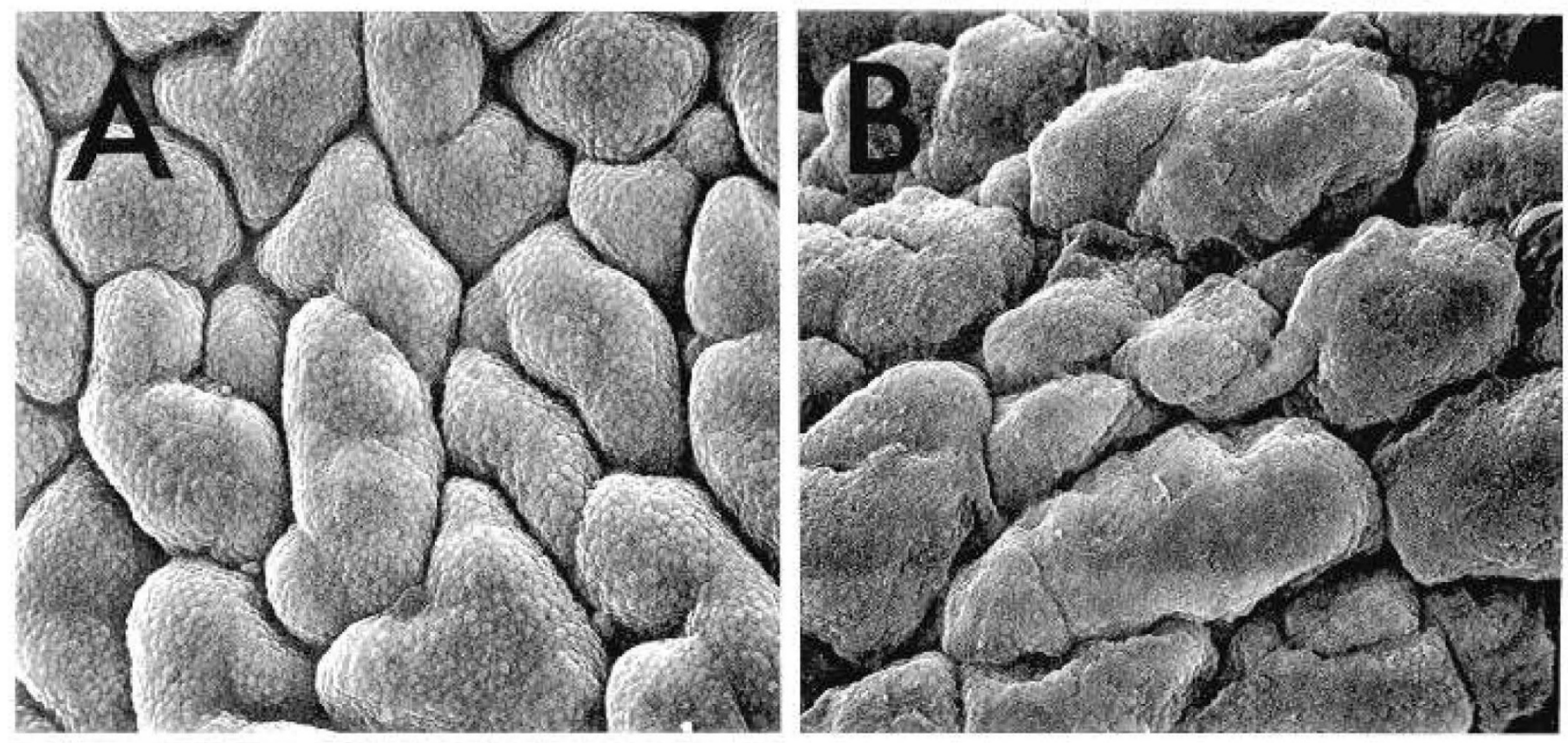

Figure 4. Scanning electron photomicrographs of the peridermal surface of the premature rat. Specimens from control $(A)$ and steroid-treated $(B)$ dams were obtained from the dorsum of pups cuthanized on the 19th d of gestation after maternal betamethasone treatment on d 17. Both specimens exhibit a "cerebriform" appearance with multiple tissue folds containing superficial granules (diameter $=1-2 \mu \mathrm{m}$ ). These granules are less distinct on the steroid-treated specimens $(B)$, and the peridermal surface appears more featureless and flattened particularly over the domes of the tissue folds. Magnification, $\times 600$.

loss). The rapid drying observed in the steroid-treated pups on d 19 and in both control and steroid-treated pups on d 20 is most consistent with the former (hydrophobic) effect. The development of a low basclinc, $\mathrm{C}_{\mathrm{bl}}$, and a low slope, $\mathrm{C}_{\mathrm{sl}}$, during continuous SEC measurement by $\mathrm{d} 20$ is consistent with the latter effect.

The effects of prenatal steroids on the developing mammal have been previously documented. Many of these studies have focused on neurologic outcome (18-25). In the human, prenatal steroids are commonly administered, specifically, to accel- erate the devclopment of lung maturity in premature infants (10). Recent studies have demonstrated an overall beneficial effect in those infants who receive steroids prenatally including lower morbidity and mortality (10). It is conceivable therefore that, in addition to accelerating lung maturity, steroids enhance the development of other organs in the body including the skin. Our findings are consistent with those of Azterbaum et al. (11, 26). In their study, using TEWL and histologic cxaminations, they showed, for the first time, that daily prenatal administration of high doses of steroids (three times the dose used in our 
study) over a 3-d period will accelerate the rate of maturation of the stratum corneum and diminish TEWL.

In our study, with the exception of pups born on gestational d 18 , prenatal exposure to steroids resulted in a lower surface hydration immediately after delivery. It is likely therefore that steroid-treated pups experienced less heat loss due to evaporation. After delivery of the pups, no significant drying of the skin was observed in either group at $\mathrm{d} 18$ and in controls at $\mathrm{d}$ 19 of gestation. A possible explanation for the failure of the premature pups' skin to dry after delivery is that the rate at which water traversed the epidermis was equal to or surpassed that at which it was lost from the skin surface. Continuous SEC readings were used therefore to generate slopes indicative of water movement across the epidermis with subsequent accumulation beneath the probe head (12). Thus, the lower slope observed in steroid-treated pups on gestational d 20 (Fig. 2C) indicates a more competent barrier and correlates well with previous reports of diminished TEWL after prenatal steroid treatment at this gestational age (11).

It should be noted, however, that the slope of continuous SEC measurements has a complicated relationship with transepidermal water movement (Figs. $2 A-C$ ). As a result, the slope of continuous SEC readings cannot be directly related to treatment group or age. On the one hand, the superficial layers of the immature skin may be saturated with water to the extent that no significant change in SEC measurements can be detected (Fig. 2A). On the other hand, a more mature epidermal barrier such as that which follows steroid treatment may be drier initially but still permeable to water at a rate sufficient to generate a significant slope (Fig. $2 B$ ). By d 20, the complete features of a mature epidermal barrier are present, viz. a low baseline SEC and a low slope during continuous SEC measurements. These findings indicate that, at the dose used in this study, it may take greater than $24 \mathrm{~h}$ for significant maturational changes to occur. Also, these findings were supported by scanning and transmission electron microscopy studies that revealed significant cornification and periderm maturation of the steroid-treated pups.

Previous studies conducted on rats and humans have demonstrated that the barrier properties of the skin are dependent on the presence of epidermal lipids (superficial and intercorneocyte) and the maturity of the stratum corneum (25). Clinical correlates can be found in the observation of increased TEWL after extraction of lipids with organic solvents or mechanical disruption of the superficial layers of the stratum corneum with adhesive tape (27). In the human, the importance of both components of barrier formation is reflected in the failure of artificial barriers to reduce the TEWL in premature infants to that found in term infants $(28-30)$. In the term human newborn, the skin surface is dry and hydrophobic $(3,4)$. These characteristics are, in part, due to a superficial lipid layer that may influence the interaction of the skin surface with exogenous water and that can be removed with common organic solvents (3).

Concerns have been raised about the potential adverse effect of steroids on the overall growth of newborn mammals. Although animal studies have documented stunted growth related to prenatal administration of steroids, no such observation has been made with respect to the premature human newborn. In the former studies $(11,18-25)$, steroids were administered at higher dosages and at greater frequency, i.e. multiple administrations. In this study, steroid-treated pups were significantly heavier than control pups on $\mathrm{d} 19$ and 20 of gestation, a result that contrasts with the findings of other investigators. A possible explanation for this finding is that a single injection of low-dose steroids, in addition to stimulating organ maturation, may stimulate total body growth of rats in utero. The large number of animals $(n=377)$ examined in this study allowed this small but significant effect to be observed.

In conclusion, we have shown that it is possible to accelerate the development of the epidermal barrier of premature rats using doses of steroids similar to those currently used in humans. Specifically, a single dose of steroids administered on d 17 of gestation accelerates maturation of the periderm and results in a more hydrophobic skin surface by gestational d 19 and 20 . These results confirm and extend the earlier report by Azterbaum et al. (11) of steroid-induced cornification and decreased TEWL in the premature rat. The clinical significance of a more mature epidermal barrier resides, in part, in a reduction in the quantity of heat lost by evaporation, either from fluid adsorbed to the skin surface or from fluid traversing the skin from deeper tissues (31). It is conceivable that a similar acceleration in the rate of skin maturation occurs in human infants who are exposed to steroids prenatally and that this phenomenon may contribute to the lower mortality and morbidity observed in these infants $(10)$.

\section{REFERENCES}

1. Phelps DL, Brown DR, Tung B, Cassady G, McClead RE, Purohit DM, Palmer EA 199128 day survival rates of 6676 neonates with birthweights of 1250 grams or less. Pediatrics 87:7-17

2. Paneth N, Wallenstein S, Kiely JL, Susser M 1982 Social class indicators and mortality in low birthweight infants. Am J Epidemiol 116:364-375

3. Okah FA, Pompas K, Wickett RR, Hoath SB 1994 The human newborn skin: effect of isopropanol on skin surface hydrophobicity. Pediatr Res 35:443-446

4. Saijo S, Tagami H 1991 Dry skin of newborn infants: functional analysis of the stratum corneum. Pediatr Dermatol 8:155-159

5. Wickett RR, Mutschelknaus JL, Hoath SB 1993 Ontogeny of water sorption- desorption in the perinatal rat. J Invest Dermatol 100:407-411

6. Hammarlund K, Sedin G 1979 Transepidermal water loss in newborn infants. IIl. Relation to gestational age. Acta Pacdiatr Scand 68:759-801

7. Sedin G, Hammarlund K, Nilsson GE, Strombcrg B, Obcrg PA 1985 Measurement of transepidermal water loss in newborn infants. Clin Perinatol 12:79-99

8. Wilson DR, Maibach HI 1980 Transepidermal water loss in vivo. Premature and term infants. Biol Neonate 37:180-185

9. Hammarlund $K$, Scdin G 1982 Transepidermal water loss in newborn infants. VI. Heat exchange with the environment in relation to gestational age. Acta Paediatr Scand 71:191-196

10. Crowley P, Chalmers I, Keirse MJNC 1990 The effects of corticosteroid administration before preterm delivery: an overview of the cvidence from controlled trials. $\mathrm{Br}$ J Obstet Gynaecol 97:11-25

11. Azterbaum M, Menon GK, Feingold KR, Williams ML 1993 Glucocorticoids accelerate fetal maturation of the cpidermal permeability barrier in the rat. J Clin Invest 91:2703-2708

12. Wickett RR, Nath V, Tanaka R, Hoath SB 1994 Usc of continuous electrical capacitance and TEWL measurement for assessing barrier function in neonatal rat skin. Skin Phamacol (in press)

13. Rosner B 1989 Table of random digits. In: Fundamentals of Biostatistics. Duxbury Press, Boston, Table 4, p 540

14. Karnovsky MJ 1965 Formaldehyde-glutaraldehyde fixation of high osmolarity for use in electron microscopy. J Cell Biol 27:137A (abstr)

15. Bozzola JJ, Russell LD 1992 Specimen preparation for transmission electron microscopy. In Electron Microscopy: Principles and Techniques for Biologists. Jones \& Bartlett, Boston, pp 16-38

16. Fisz M 1963 Chebyschev law of large numbers. In: Probability Theory of Mathematical Statistics. Wiley, New York, pp 175-249

17. Chang JC, Dale BA, Brown BE, Elias PM 1986 Histochemical and morphologic studies on mammalian epidermal peridermal granules. Br J Dermatol 114:431-440 
18. Uno H, Lohmiller L, Thieme C, Kemnitz JW, Engle, MJ, Roecker EB, Farrell PM 1990 Brain damage induced by prenatal exposure to dexamethasone in fetal rhesus macaques. I. Ilippocampus. Dev Brain Res 53:157-167

19. Johnson JWC, Mitzner W, London WT, Palmer AE, Scott R 1979 Betamethasone and the rhesus fetus: multisystemic effects. Am J Obstet Gynecol 133:677:684

20. Slotkin TA, Lappi SE, McCook EC, Tayyeb MI, Eylers JP, Seidler F\} 1992 Glucocorticoids and the development of neuronal function: effects of prenatal dexamethasone exposure on central noradrenergic activity. Biol Neonate 61:326-336

21. Carlos RQ, Seidler FJ, Slotkin. TA 1992 Fetal dexamethasone exposure alter macromolecular characteristics of rat brain development: a critical period for regionally selective alterations? Teratology 46:45-59

22. Carlos RQ, Seidler FJ, Slotkin TA 1991 Fetal dexamethasone exposure sensitizes neonatal rat brain to hypoxia; cffects on protein and DNA synthesis. Dev Brain Res 64:161-166

23. Carlos RQ, Seidier FJ, Lappi SE, Siotkin TA 1991 Fetal dexamethasone exposure affects basal omithine decarboxylase activity in developing brain regions and alters acute responses to hypoxia and maternal separation. Biol Neonate 59:69-77

24. Bian X, Seidler FJ, Slotkin TA 1993 Fetal dexamethasone exposure interferes with establishment of cardiac noradrenergic innervation and sympathetic activity. Teratology $47: 109-117$
25. Secard RE 1992 Dexamethasone induces a transient relative cardiomegaly in neonatal rats. Pediatr Res 31:359-363

26. Azterbaum M, Menon GK, Feingold KR, Williams ML 1992 Ontogeny of epidermal barrier to water loss in the rat: correlation of function with stratum corneum lipids. Pediatr Res 31:308-317

27. Tagami H 1989 Impedance measurement for evaluation of the hydration state of the skin surface. In: Leveque JL (cd) Cutaneous Investigation in Health and Disease-Noninvasive Methods and Instrumentation. Marcel Dekker, New York, pp $79-111$

28. Vernon HJ, Lane AT, Wischerath LJ, Davis JM, Menegus MA 1990 Semipermeable dressing and transepidermal water loss in premature infants. Pcdiatrics 86:357-362.

29. Knauth A, Gordin M, McNelis W, Baumgart S 1989 Semipermeable polyurcthanc membrane as an artificial skin for the premature neonate. Pcdiatrics 83:945-950

30. Bustamante SA, Steslow J 1989 Use of a transparent adhesive dressing in very low birthweight infants. J Perinatol 9:165-169.

31. Chameides $\mathbf{L}$ and The AHA/AAP Neonatal Resuscitation Steering Committee 1990 Textbook of Neonatal Resuscitation. American Heart Association and American Academy of Pediatrics, Elk Grove Village, IL, pp 2-9 